\title{
Pobreza, Migración Académica y Estereotipos de Género en la Educación Superior, la Ciencia y la Tecnología
}

\section{Poverty, Academic Migration and Gender Stereotypes in Higher Education, Science and Technology}

\author{
Roger J. González González * \\ Edith J. Cisneros-Cohernour \\ Galo E. López Gamboa
}

Universidad Autónoma de Yucatán, México

\begin{abstract}
Esta investigación tuvo como propósito comprender los retos, las experiencias y las cuestiones críticas de una joven maya durante su incursión en la educación superior tecnológica, la ciencia y la tecnología en el sureste de México. La investigación siguió un diseño por estudio de caso cualitativo; el caso lo constituye Flor, una joven maya del estado de Yucatán que cursa la educación superior y participa en actividades relacionadas con el fomento a la investigación científica y tecnológica. Entre los resultados, se encontró que Flor enfrenta retos y cuestiones críticas vinculados a temas como la pobreza, la presión académica y expectativas familiares, la migración y acceso a la educación, cuestiones de género en la familia e ingenierías "para mujeres", la calidad de la educación rural, así como la influencia de modelos para la formación académica y en investigación. Los resultados pueden ser tomados como base para futuras investigaciones, que estudien las cuestiones críticas que emergen con poblaciones más amplias de jóvenes originarios de comunidades rurales en Yucatán, particularmente aquellos estudiantes que cursan carreras relacionadas con la ciencia y tecnología en la región maya de México.
\end{abstract}

Descriptores: Educación superior; Ciencia; Tecnología; Ingeniería; Género.

The purpose of this research was to understand the challenges, experiences and critical issues of a young Mayan woman during her foray into technological higher education, science and technology in southeastern Mexico. The research followed a qualitative case study design; the case is constituted by Flor, a young Mayan from the state of Yucatan who is studying higher education and participates in activities related to the promotion of scientific and technological research. Among the results, it was found that Flor faces challenges and critical issues related to issues such as poverty, academic pressure and family expectations, migration and access to education, gender issues in the family and engineering "for women", the quality of rural education, as well as the influence of models for academic and research training. The results can be taken as the basis for future research that studies the critical issues that emerge with larger populations of young people from rural communities in Yucatán, particularly those students pursuing science and technology-related careers in the Mayan region of Mexico.

Keywords: Higher education; Science; Technology; Engineering; Gender.

*Contacto: rogr.gonzalez@gmail.com

ISSN: 0718-7378

www.rinace.net/rlei/
Recibido: $\quad 18 / 06 / 2020$

$1^{\text {a }}$ Evaluación: 30/07/2020

Aceptado: 25/09/2020 


\section{Introducción}

Esta investigación forma parte de la primera etapa de un estudio más amplio, que lleva por nombre Justicia social e inequidad educativa en la formación científica y tecnológica de jóvenes de comunidades rurales en Yucatán, el cual se llevó a cabo de 2017 a 2020 con apoyo del Consejo Nacional de Ciencia y Tecnología (CONACYT). El estudio completo se divide en dos etapas, de las cuales el presente es el segundo estudio de casos de los dos que integran la primera fase de la investigación (González González y CisnerosCohernour, 2020).

Históricamente, las mujeres representan uno de los principales grupos marginados por las sociedades (Kumar y Yadav, 2018), pues a pesar de que la participación de la mujer en el ámbito de la educación contribuye al desarrollo de las sociedades, existe un serio rezago y en consecuencia la generación de disparidades en el acceso que las niñas, las jóvenes y las mujeres en general tienen a la educación (Di Cesare, 2014); siendo esta una de las principales formas de violencia y marginación que viven desde edades tempranas, recordando que en la región de América Latina, México y Centro América constituyen las zonas donde se presentan mayores índices de violencia de género contra las mujeres (ONU Mujeres, 2017).

En sectores como la educación superior, al ciencia y la tecnología se manifiestan múltiples situaciones que permiten abordar la desigualdad, inequidad y en consecuencia la violencia contra las mujeres en México. Si bien la presencia de las mujeres en la educación superior se ha incrementado e incluso prácticamente igualado en matrícula al número de varones en este nivel educativo, siguen habiendo serias diferencias en carreras profesionales vinculadas a la ciencia, la tecnología y las ingenierías en México (Asociación Nacional de Universidades e Instituciones de Educación Superior [ANUIES], 2019; Izquierdo y Atristan, 2019; Jiménez Izquierdo, Magaña Medina y Valdés Cuervo, 2019; Remes y Cervera, 2016; Rodríguez, 2008).

Muestra de la desigualdad entre hombres y mujeres en sectores específicos como la educación superior, la ciencia y la tecnología es el Sistema Nacional de Investigadores (SNI) en México, instrumento de política pública que tiene como objetivo "reconocer, como resultado de la evaluación, la calidad de la investigación científica y tecnológica que se producen en el país o por mexicanos en el extranjero" (CONACYT, 2017, p. 2); pues según los últimos datos reportados por el CONACYT (2018), de los 28,633 investigadores que conforman el SNI, el 63\% $(17,983)$ son hombres, existiendo una marcada diferencia con relación a las investigadoras que pertenecen al Sistema, quienes representan apenas el $37 \%(10,650)$ del total, estos datos se pueden apreciar en la figura 1.

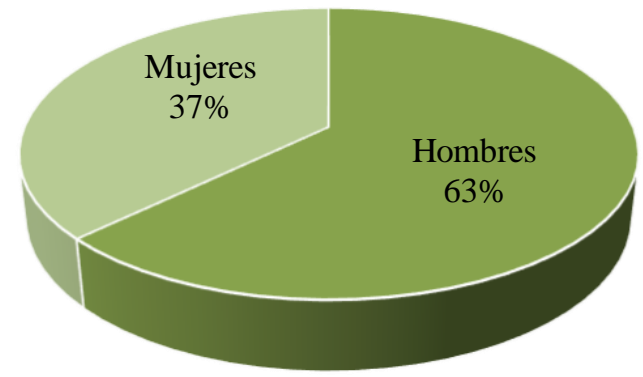

Figura 1. Distribución de mujeres y hombres en el Sistema Nacional de Investigadores Fuente: Elaboración propia. 
A pesar de que el número de hombres casi duplica al de mujeres en el Sistema Nacional de Investigadores, las diferencias en cuanto a la participación y el logro de las académicas mexicanas en este sector son aún más evidentes si el análisis se realiza por nivel alcanzado en el Sistema. Esto se aprecia a través de las evaluaciones correspondientes, en las que los participantes en el SNI pueden alcanzar alguno de los siguientes niveles: Candidato(a) a Investigador(a) Nacional, Investigador(a) Nacional nivel 1, Investigador(a) Nacional nivel 2 e Investigador(a) Nacional nivel 3. Es importante tener en consideración que adicionalmente, dependiendo de la edad del investigador y del tiempo perteneciendo al SNI, existe un rubro para los investigadores y las investigadoras eméritos.

Como se ha comentado, las diferencias son más significativas por nivel alcanzado en el SNI, ya que como se ilustra en la figura 2, en el nivel inicial de Candidato(a) a Investigador(a) Nacional, los hombres $(3,680)$ superan a las mujeres $(2,868)$ y en cuanto al SNI 1, el número de hombres $(9,378)$ casi duplica al de mujeres $(5,767)$. La diferencia se hace aún más grande en los niveles superiores del Sistema, pues como se aprecia en la figura, en el nivel 2 del SNI hay más del doble de hombres $(3,084)$ que de mujeres $(1,488)$; mientras que en el nivel más elevado, el SNI 3, existen tres veces más hombres $(1,841)$ que mujeres (527).

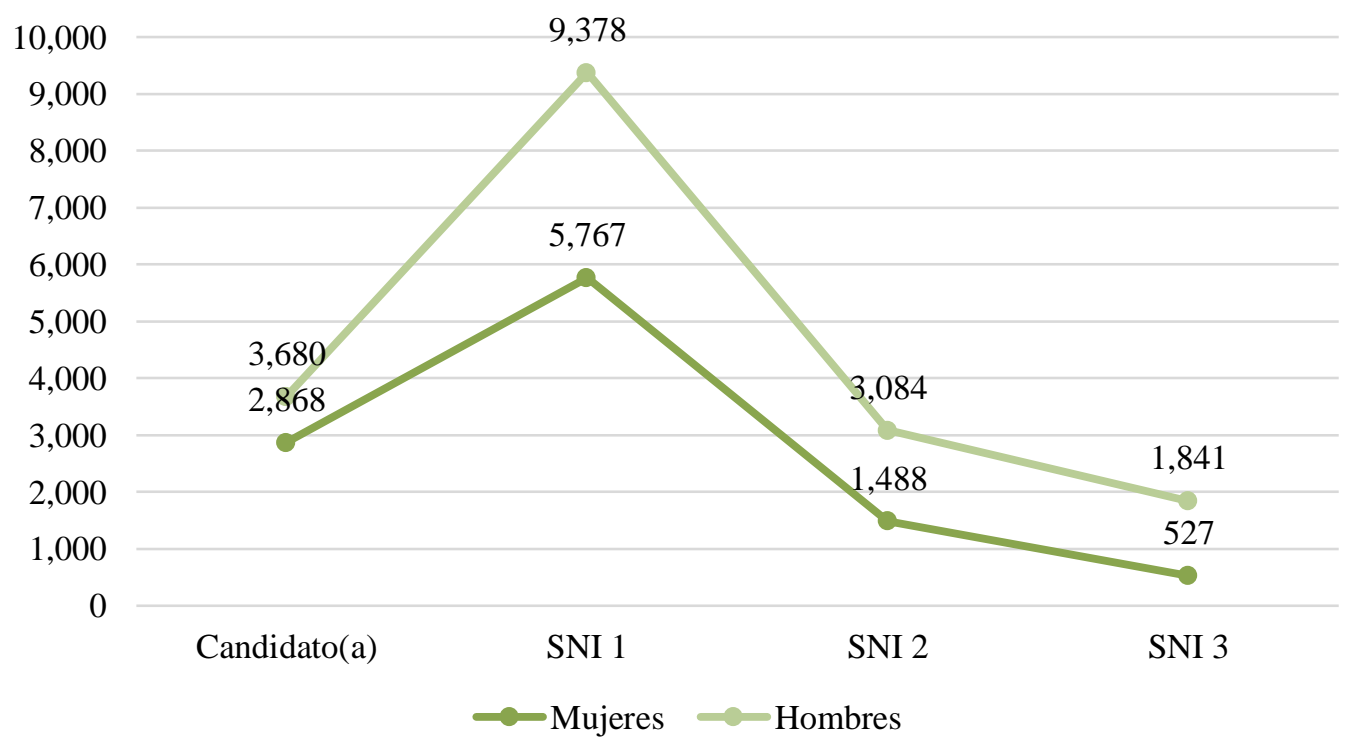

Figura 2. Distribución de mujeres y hombres en el Sistema Nacional de Investigadores por nivel

Fuente: Elaboración propia.

Estos datos son claros en cuanto a la desigualdad que hay entre hombres y mujeres en sectores como la educación superior, la ciencia y la tecnología en México; sin embargo existen regiones en el país que se caracterizan por el rezago científico y tecnológico, tal es el caso de la región maya de México, y particularmente del estado de Yucatán, que junto con estados como Oaxaca y Chiapas acumulan el 42.6\% del total de indígenas en México (Instituto Nacional de Estadística y Geografía [INEGI], 2015).

En Yucatán, las diferencias en la participación de hombres y mujeres en el Sistema Nacional de Investigadores se agudizan en los niveles superiores, pues si bien en el nivel candidato la participación es totalmente igualitaria (72 investigadores y 72 
investigadoras), en el nivel 1 el número de hombres (247) es casi el doble que el de mujeres (134). Como se aprecia en la figura 3 la tendencia se mantiene en el nivel 2, en el que se encuentra a 57 hombres y 30 mujeres, sin embargo en el nivel 3 hay cerca de cinco veces más hombres (39) que mujeres (8).

Además de estos datos, es necesario tener en cuenta que de las 244 mujeres que en Yucatán pertenecen al Sistema Nacional de Investigadores, el 32.7\% se desempeña en áreas como las humanidades, ciencias de la conducta y ciencias sociales; viendo reducida su participación en áreas como ingenierías, matemáticas, biotecnología, entre otras disciplinas tradicionalmente clasificadas como ciencias duras.

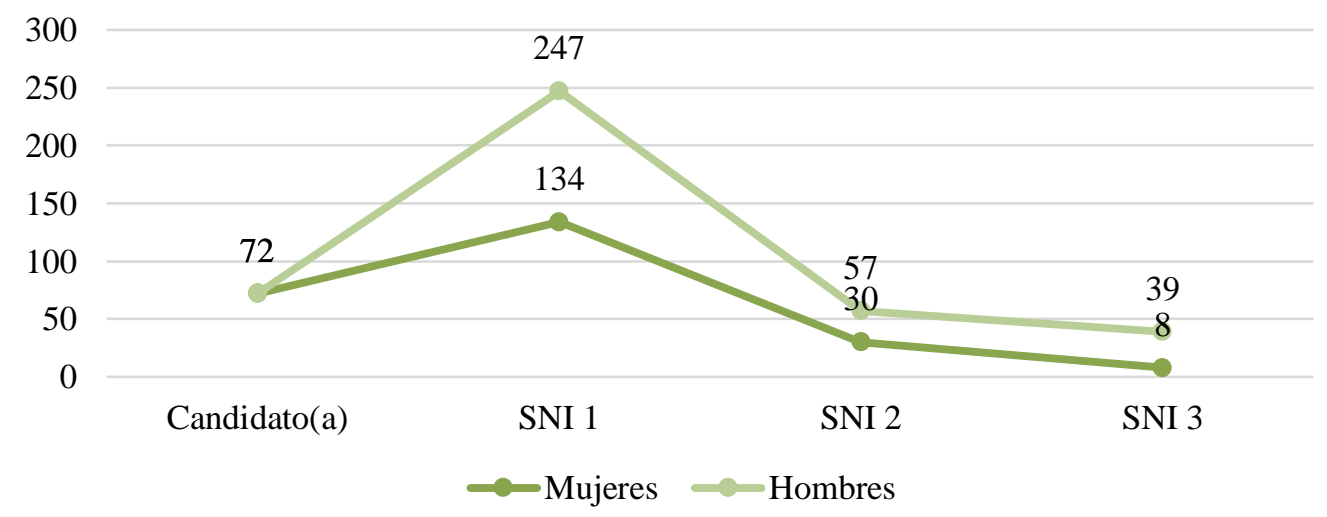

Figura 3. Distribución de mujeres y hombres de Yucatán en el Sistema Nacional de Investigadores por nivel Fuente: Elaboración propia.

Para el caso de Yucatán, el 65.4\% de la población se reconoce como personas indígenas, siendo la segunda entidad con mayor población indígena en México (INEGI, 2015), y se enfrenta a múltiples problemáticas sociales, económicas y culturales como la pobreza, la marginación, el rezago educativo en niñas y niños indígenas (Mijangos, Canto y Cisneros, 2009), así como la violencia de género en niñas y adolescentes en el estado (Consejo Estatal de Población [COESPO], 2020).

En cuanto a la calidad de la educación en Yucatán, es necesario señalar que el 30.1\% de la población en las comunidades mayas del estado presenta carencias por rezago educativo, mientras el $75.9 \%$ de la población de 15 años y más con primaria incompleta son indígenas, ubicando a Yucatán como el estado con mayor rezago educativo para la población indígena en todo el país (Gobierno del estado de Yucatán, 2019).

A pesar de los esfuerzos por alcanzar la paridad de género en la asistencia escolar, la brecha sigue siendo amplia en países de bajos ingresos como México, especialmente en las áreas rurales (UNESCO, 2003). Tal es el caso de las jóvenes indígenas de Yucatán, que tienen un limitado acceso a un sistema educativo de calidad e incluyente (Gobierno del Estado de Yucatán, 2019), aunado al hecho de que se ha constatado que en México, la participación de la mujer en el ámbito de la investigación científica es limitada (Cárdenas, 2015; Rodríguez, 2016). 


\section{Método}

Es así que se considera necesario explorar las situaciones que enfrentan las jóvenes de Yucatán durante su incursión en ámbitos como la educación superior, la ciencia y la tecnología. Por ello se plantea como propósito de esta investigación comprender los retos, las experiencias y las cuestiones críticas de una joven maya durante su incursión en la educación superior tecnológica, la ciencia y la tecnología. El caso está representado por Flor, una joven maya de una comunidad rural ubicada en la parte poniente del estado de Yucatán, quien enfrenta retos durante su formación académica y su vida.

Esta investigación sigue un diseño por estudio de caso cualitativo e instrumental, ya que este diseño ayuda a tener una comprensión general de una situación a través del estudio de casos particulares, así como a conocer las cuestiones críticas del problema (Stake, 1998). Este es un estudio cualitativo, pues las bondades de este método contribuyen a entender y caracterizar un fenómeno a través diferentes herramientas, diferentes voces, perspectivas y puntos de vista que permiten comprender la realidad más que objetivarla, pues recordemos que la realidad objetiva nunca puede ser capturada (Denzin y Lincoln, 2005).

\section{Caracterización del caso}

Para lograr una mejor comprensión de los fenómenos y temas críticos en una investigación, es necesario elegir correctamente el caso (Patton, 2015; Yin, 1989 en Denzin y Lincoln, 2005). Por ello es que el estudio se centra en Flor, los contextos en los que interactúa y las múltiples voces que ayudan a la triangulación de los datos, así como a la reconstrucción de la realidad.

Flor es una joven de 20 años originaria de San Isidro, una comunidad maya y rural ubicada $65 \mathrm{~km}$ al oeste de la ciudad de Mérida, capital del estado de Yucatán en el sureste de México. La joven se encuentra actualmente cursando la Ingeniería bioquímica en la Universidad Tecnológica de Mérida, y tuvo que migrar a la capital del estado para poder realizar sus estudios de nivel superior, regresando los sábados y domingos a su comunidad.

Desde los 11 años, Flor ha tenido un fuerte interés por la ciencia y la tecnología, participando en actividades como el programa Jóvenes Talentos del CONACYT, olimpiadas del conocimiento, concursos de ciencia, entre otras. Adicionalmente, desde los 15 años viaja a otras comunidades para realizar sus estudios de educación formal.

\section{Contextos}

Flor interactúa en múltiples contextos, tanto en zonas rurales como urbanas, entre los que se encuentran la ciudad de Mérida, la comunidad de San Isidro y la Universidad Tecnológica de Mérida. Las observaciones e interacción se llevaron a cabo en los contextos antes mencionados, así como en los trayectos a la escuela, la comunidad y las actividades de fomento a la investigación científica y tecnológica en las que la joven se involucra.

Vale la pena señalar que los contextos familiar y escolar fueron los que proveyeron de mayor información a la investigación. Adicionalmente se realizaron sondeos y pláticas informales con personas de la comunidad de Flor, esta información contribuyó a entender aspectos como la dinámica comunitaria y los proceso educativos en San Isidro. 


\section{Participantes}

En el estudio participaron personas clave en la trayectoria académica y en la vida de Flor, quienes proporcionaron información a partir de la cual se construyeron categorías y emergieron las cuestiones críticas del caso. A continuación, se describe a los participantes en el estudio de caso de Flor:

- Familia. Se realizaron entrevistas con la mamá, el papá, el hermano y el novio de Flor. A través de esta información se pudo reconstruir parte de la trayectoria personal y académica de la joven.

- Maestros. Se entrevistó a profesores de la Universidad Tecnológica de Mérida, lo cual permitió confirmar cuestiones críticas que fueron emergiendo con relación a la calidad de la educación superior en el estado, particularmente cuestiones vinculadas al género y desigualdades en las carreras profesionales que los jóvenes y las jóvenes cursan en las instituciones de educación superior tecnológica de Yucatán.

- Amigas y compañeras. Se entrevistó a compañeras de la escuela de Flor, quienes tienen una percepción similar a la de la joven en cuanto a la participación de la mujer en las ingenierías, así como el ambiente que existe en la Universidad Tecnológica de Mérida y su dinámica.

Adicionalmente se entrevistó a dos de las amigas de Flor, quienes proporcionaron información acerca de la calidad de la educación y los docentes de su comunidad de origen.

- Personas de la comunidad. Se tuvo interacción y pláticas informales con personas de la comunidad de Flor, esto permitió entender parte de la dinámica y tradiciones de la comunidad.

Para la colecta de datos se recurrió a múltiples técnicas como son el análisis documental, las entrevistas grupales, entrevistas a profundidad, entrevistas semiestructuradas y la observación, técnica básica en todo el proyecto de investigación.

\section{Técnicas para la colecta de datos}

El estudio inició en diciembre de 2017 y concluyó en abril de 2019, tiempo en el que se pudo acompañar a Flor en sus contextos de interacción, conviviendo con personas que son significativas tanto en su trayectoria académica como en su vida personal.

Cuadro 1. Participantes y entrevistas que se llevaron a cabo como parte del estudio de caso de Flor

\begin{tabular}{lcc}
\hline & ENTREVISTAS (N) & PARTICIPANTES (N) \\
\hline Flor & 9 & 1 \\
Madre y padre & 3 & 2 \\
Hermano & 2 & 1 \\
Novio & 3 & 1 \\
Profesores-investigadores & 2 & 2 \\
Compañeras de la universidad & 2 & 2 \\
Amigas de la comunidad & 2 & 2 \\
\hline Total & 23 & 11 \\
\hline
\end{tabular}

Fuente: Elaboración propia.

Como se aprecia en el cuadro 1, se realizaron 23 entrevistas distribuidas entre Flor y 10 personas con las que la joven más interactúa, quienes contribuyeron a la comprensión de 
las situaciones que vive Flor durante su formación. Es importante mencionar que las entrevistas se llevaron a cabo en diferentes modalidades como son entrevistas a profundidad, entrevistas semi-estructuradas y entrevistas grupales.

Adicionalmente, la observación fue la técnica básica a lo largo del proyecto, permitiendo profundizar en el análisis de las cuestiones críticas, ya que de acuerdo con Guba y Lincoln (1981), en situaciones donde los valores, creencias, actitudes y experiencias tienen una elevada presencia en el fenómeno estudiado, la observación es el instrumento más sofisticado de investigación, permitiendo al investigador mirar, ver, escuchar, preguntar, sondear, analizar y organizar su experiencia.

\section{Triangulación del estudio}

En esta investigación el proceso de triangulación se realizó a través de fuentes de información, pues en consistencia con Campbell y Fiske (1959 en Stake 1998) "para conseguir constructos útiles e hipotéticamente realistas en una ciencia se requieren métodos múltiples que se centren en el diagnóstico del mismo constructo desde puntos de observación independientes, mediante una especie de triangulación” (p. 99).

Triangulamos nuestros datos para aumentar la confianza de que hemos interpretado correctamente cómo funcionan las cosas. A veces nuestros puntos de vista son defectuosos porque son demasiado simplistas. La triangulación nos ayuda a reconocer que las cosas necesitan más explicación de lo que pensamos al principio (Stake, 2010)

\section{Resultados}

El estudio de caso de Flor es valioso pues presenta los retos y las experiencias que vive una joven proveniente de una comunidad rural durante su formación académica y en investigación, cuestiones que no pretendemos sean generalizables pero sí pueden servir como base para entender de manera exploratoria cómo viven las jóvenes de comunidades rurales de Yucatán su formación académica en el ámbito de la ciencia y tecnología.

Toda la información recaba a través de las entrevistas fue grabada con el consentimiento de los participantes, de igual forma el registro que se llevó a través del diario de campo permitió establecer constructos claves con base en las observaciones realizadas.

Para el análisis de los datos se recurrió a la técnica de diagramas de afinidad, que permite agrupar y categorizar de forma natural los datos, emergiendo temas generales y categorías que se relacionan entre sí (Kawakita, 1977). Como se ilustra en la Figura 4, en el estudio emergieron seis categorías, las cuales son: 1) Pobreza, 2) Presión académica y expectativas familiares, 3) Migración y acceso a la educación, 4) Género en la familia e ingenierías "para mujeres”, 5) Calidad de la educación rural y 6) Modelos para la formación académica y en investigación.

A continuación se describen las categorías que emergieron en el estudio, presentando situaciones y fragmentos del discurso de los participantes, lo cual permite ilustrar las cuestiones críticas más significativas en la trayectoria académica y la vida de Flor. 


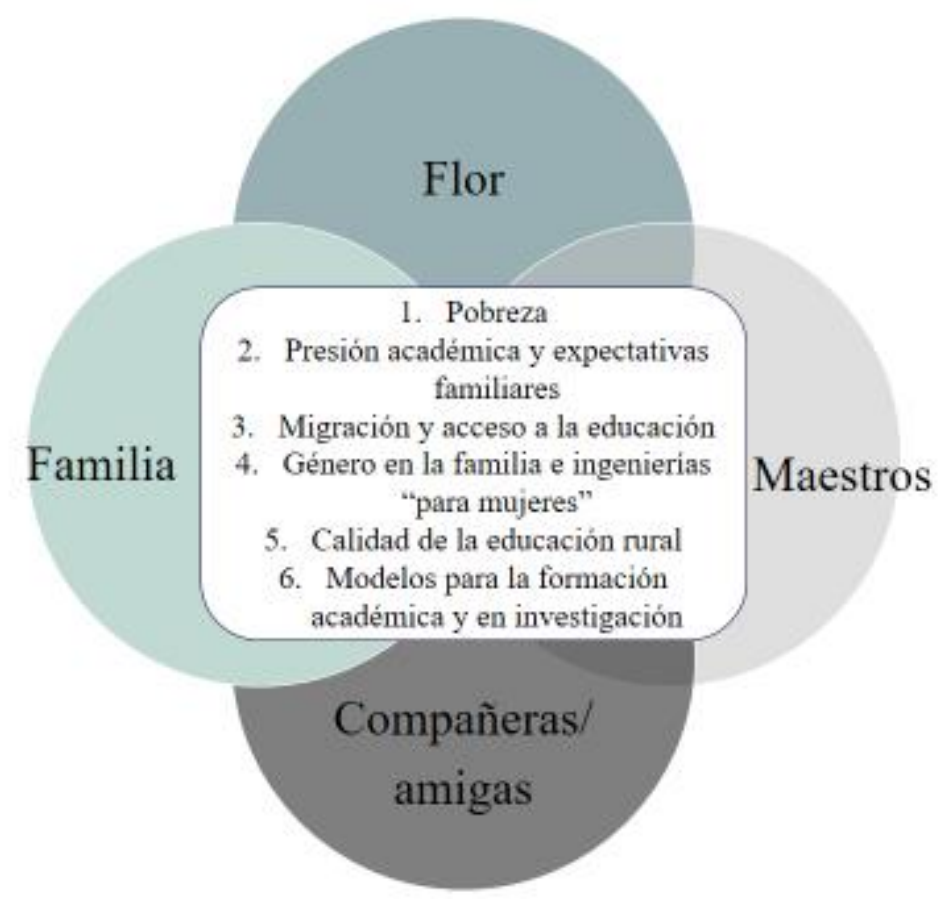

Figura 4. Categorías que emergen del estudio de caso de Flor Fuente: Elaboración propia.

\subsection{Pobreza}

A través de las observaciones, la interacción con Flor, su familia, amigos y miembros de su comunidad, emergió la categoría Pobreza, tema enraizado en la vida diaria de los participantes, su discurso e incluso en su interacción con otras personas de la comunidad. Con relación a este fenómeno que afecta de manera significativa a las familias mayas de Yucatán, el padre de Flor relata:

$$
\begin{aligned}
& \text {... soy empleado en una escuela en la cual pues la verdad no ganamos lo suficiente que } \\
& \text { se diga... } \\
& \text {... yo no me dedico solamente a lo que es aqui en la escuela, yo tengo mi moto taxi, me } \\
& \text { pongo a hacer otros trabajitos para poder subsistir y ayudar mucho a Flor... }
\end{aligned}
$$

A pesar de la situación económica en la que se encuentra la familia, con la finalidad de que Flor cursara la educación superior, la madre de la joven comenta que en su momento le propuso migrar al centro del país para poder estudiar una licenciatura:

...le comenté a mi hija hace como dos años, tres años, le digo cuando terminó la COBAY, le digo ¿̇hija por qué no presentas examen para Chapingo y te vas?, porque papá a veces no tiene, le digo, mejor ahí te vas becada, te vas...

Este tipo de acontecimientos son complejos para la familia pues son muy unidos, sin embargo la pobreza en la que viven los ha hecho considerar opciones como la migración de los hijos, así como las consecuencias que esto conlleve para poder continuar con sus estudios por medio de becas y apoyos.

Vale la pena señalar que la situación económica en la que vive la familia de Flor, según comentan y se observa, es una constante en la comunidad, fenómeno que influye de manera significativa en la vida de los habitantes de San Isidro y la percepción que tienen de sí 
mismos con relación la gente que vive en la capital del estado. Al respecto Don Santiago, de 52 años y quien toda su vida ha habitado en San Isidro comenta:

...la gente humilde que ha salido adelante lo valora más, no la gente de dinero de Mérida que van...en la escuela.

La incursión de Flor en la educación superior, la ciencia y la tecnología han representado un gran esfuerzo para la familia, sin embargo son conscientes de la importancia que tiene apoyar a la joven en estos ámbitos. El padre ilustra el impacto en la economía familiar y el apoyo como familia a la formación de Flor:

...ella estuvo viajando diario, sabes qué yo le decía, pues sabes una cosa yo ahí veía, el último camión que llegaba, alcanzaba para que yo en Umàn (comunidad por la que el transporte transita de la ciudad de Mérida a la comunidad de San Isidro) la iba a buscar en mi cochecito, diarios por un mes.

Le digo si multiplicas $\$ 150$ por un mes, saca tu cuenta cuánto es, ya con todo, haciendo esfuerzo diario la iba yo a buscar, y a veces son 10:30 de la noche porque ya pasó el último (camión), 10:30, 11:00 de la noche, a veces estoy viniendo a las 12:00 de la noche con ella, durmiendo estoy viniendo.

En este breve fragmento se encuentran algunos de los principales retos que envuelven el caso de Flor, y que dan origen a las cuestiones críticas que enfrenta la joven.

\subsection{Consecuencias de Flor en la ciencia: Presión académica y expectativas familiares}

A través del acompañamiento de Flor, las observaciones y la interacción con su familia, se pudo encontrar que muchas de las decisiones, actividades y circunstancias que vive, tanto en el ámbito personal como académico han estado determinadas por la presión familiar y las expectativas que tienen acerca del futuro de la joven. Con relación al apoyo que la familia le ha dado a Flor durante su formación académica, y en consecuencia las expectativas y la presión que se han generado, el padre precisa:

\section{... Se le ha apoyado desde, pues desde, hasta donde está ahorita porque es como yo le digo a ella, también ella tiene que dar de su parte para que se pueda aprovechar lo poco o mucho que le damos, igual con mi esposa. \\ ...Es lo que le digo a ella, estás estudiando en Mérida, quédate, porque pues es un sacrificio grandísimo.}

Por otro lado, se considera que parte de la presión ejercida por los padres hacia la joven se debe a las oportunidades y al éxito, que según comentan, ellos no tuvieron en su momento. A continuación se presentan fragmentos del discurso de la madre de la joven, que permite ejemplificar el tema de la Presión académica y expectativas familiares:

$$
\begin{aligned}
& \text {...por eso a veces yo le digo a mi hija, hija estudia y es lógico que vas a tener novio, } \\
& \text { pero... ¿̇quieres quedarte como yo? } \\
& \text {...Por eso le digo a Flor, estudia. Sé que le habla el muchacho y todo, pero primero } \\
& \text { está la escuela...Ella sabe cómo quiere terminar, la verdad, le digo... }
\end{aligned}
$$

La presión familiar y las expectativas académicas de los padres con relación al futuro de Flor han influido en aspectos básicos como su elección vocacional. Flor comenta que originalmente ella tenía la intensión de cursar la Licenciatura en Químico Farmacéutico Biólogo que oferta la Universidad Autónoma de Yucatán, sin embargo relata que la principal razón por la que decidió estudiar Ingeniería Bioquímica fue por su padre, al respecto comenta: 
...Mi papá me decía, no estudies una licenciatura, estudia una ingeniería y siempre tuve lo de mi papá, de no estudies eso, no estudies eso... y ya no presenté para QFB [Químico Farmacéutico Biólogo].

Es evidente la influencia del padre en la elección vocacional de Flor, no solo por los comentarios de la joven sino que, a través de la interacción con él, en las pláticas y entrevistas se pudo notar su preferencia por el ámbito de las ingenierías y su desagrado por las carreras que se ofertan en la modalidad de licenciatura. En una plática con el padre, comentó:

... me da gusto que estudies para ingeniera, porque aquí como trabajo con otros chamacos que pues están empezando, ;se sienten pesadotes!, por eso le digo, a veces vulgarmente como le digo, licenciado cualquier perro es, ingeniero no, le digo [risas del padre].

En otra ocasión, el padre realizó otro comentario acerca del tema:

...Es que hay chamacos nuevos de acá, licenciados que me molestan, se sienten muy altos los pobrecitos, pena me dan le digo.

Es necesario mencionar que parte del rechazo del padre de Flor por las licenciaturas y su influencia en la elección profesional de la joven se debe a los antecedentes académicos del padre, quien siempre ha tenido interés por las ingenierías, por lo cual comenta que durante su juventud tuvo intenciones de cursar una ingeniería en la Universidad Tecnológica de Mérida. Al respecto precisa:

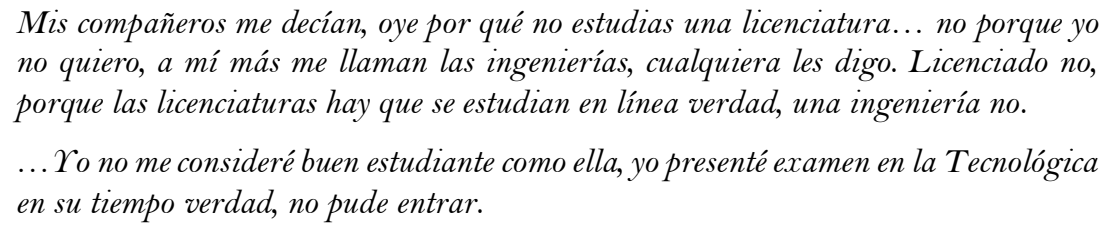

Parte de esta postura, así como la presión académica y expectativas familiares se vinculan a temas de género, lo cual se explica más adelante en un apartado en el que se explora el tema de género durante la educación superior de Flor.

\subsection{Migración y acceso a la educación}

Como se ha encontrado en otros estudios realizados en la zona maya de México (González González y Cisneros-Cohernour, 2020), la falta de acceso a la educación conlleva a la migración de los jóvenes de comunidades rurales de Yucatán. La migración por acceso a la educación representa uno de los principales retos que Flor ha enfrentado, como relata el padre:

...ella viaja mucho, está estudiando y pues, le tengo hecho una pequeña casita en Mérida, le digo aunque te quedes porque es peligroso viajar diario, por eso hago mis esfuerzos para hacer las cosas.

Por otro lado la madre de Flor comenta que además de que la joven tuvo que migrar a la ciudad de Mérida para cursar la educación superior, parte de la vida de Flor se ha caracterizado por los constantes viajes a la ciudad de Mérida y a una comunidad vecina en la que cursó el bachillerato. Al respecto la madre relata:

...como estudiaba en San Sebastián [Como ya se ha comentado, Flor es originaria de San Isidro], tenía que levantarse a las 5:00 a.m. para viajar a San Sebastián; después regresaba, a la 1:10 p.m. salía y a la 1:40 p.m. ya estaba regresando aquí. Solo se cambiaba y se iba al sitio de taxis porque a las tres estaba saliendo el camión para irse, y cuando estuvo en el Hideyo [Centro público de investigación ubicado en la ciudad de Mérida] no fue tanto problema porque estaba cerca, pero en el CICX 
[Otro centro de investigación de la ciudad] fue horrible, porque llegaba ahi a las 4:30 o 5:00 p.m. y la Doctora (con la que trabajaba) ya se iba.

Los constantes viajes, distancias y dificultades en el acceso a la educación han influido incluso en la carrera profesional que Flor eligió, pues al respecto la joven comenta:

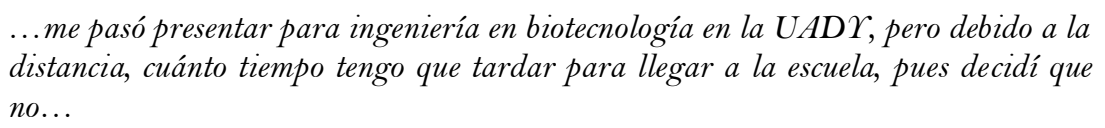

El hecho de que la carrera de Ingeniería en Biotecnología, ofertada por la Universidad Autónoma de Yucatán, se impartiera en una escuela más lejana para Flor, influyó en su decisión de estudiar Ingeniería Bioquímica en la Universidad Tecnológica de Mérida.

Flor comenta que el no vivir en Mérida influye significativamente en su ritmo de vida y el hecho de tener que migrar a la capital del estado para cursar la educación superior, pues comenta:

...ya no tengo tiempo de hacer nada...con eso de la escuela, sí influye mucho que yo no viva en Mérida porque son dos horas de viaje...entonces llego, como y tengo que hacer mi tarea y ya no tengo tiempo de hacer otras cosas...

Finalmente, se considera que este fenómeno ha generado una perspectiva negativa por parte de Flor hacia las personas, y particularmente los jóvenes que viven en la ciudad de Mérida, pues comenta que para ellos participar en actividades de fomento a la investigación como el programa Jóvenes Talentos es más fácil. Con relación a este tema la joven comentó:

\begin{abstract}
... los que viven en Mérida no lo sufren, o sea son cosas que si yo viviera en Mérida, pues fácilmente iba a Jóvenes Talentos todo el día, entonces todas esas cosas que tú como pueblo no puedes hacer, que los de Mérida sí pueden... tenía compañeros [De la ciudad de Mérida] que iban saliendo de la escuela, 1:30 de la tardes ya están en el laboratorio y 7:00 de la noche se están quitando, y yo no, llego a las 3:00 y a las 5:00 me tengo que quitar porque a las 7:00 tengo qué llegar aquí [a San Isidro], entonces son tiempos muy limitados...
\end{abstract}

El discurso de Flor denota las diferencias que percibe entre los jóvenes de comunidades rurales y los de la ciudad de Mérida para ingresar a la educación superior y participar en actividades de fomento a la investigación científica y tecnológica, pues según la participante, para estos últimos es más fácil involucrarse en las actividades que se desarrollan en los núcleos urbanos de la región.

\title{
3.4. Género en la familia e ingenierías "para mujeres"
}

En el estudio de caso de Flor emergió el tema de la desigualdad de género en ámbitos como la educación superior, la ciencia y la tecnología. A continuación se presentan fragmentos de entrevistas, pláticas y observaciones a través de las que se pudo identificar que Flor ha vivido episodios de desigualdad de género por parte de su familia y maestros.

Parte de las desigualdades de género a las que Flor se ha enfrentado tienen origen en su propia familia, pues la madre asume la responsabilidad de educar y los procesos de formación académica de los hijos como un deber de ella, en el que su esposo la apoya. Se ilustra esta cuestión a través de la siguiente viñeta:

$$
\begin{aligned}
& \text {...él me dice [el padre de Flor], no ha te ayudo con su pasaje... bueno pues el pasaje, } \\
& \text { es mucho diario... }
\end{aligned}
$$

Al ser la madre la responsable de la educación de los hijos, en ocasiones tiene que viajar todos los días a la ciudad de Mérida y de regreso a su comunidad para cumplir con este 
deber. Llega a Mérida por las tardes para acompañar a Flor y dormir con ella y al día siguiente por la mañana, regresa lo más temprano posible a San Isidro. La madre narra parte de esta situación y el hecho de hacerse cargo de la educación de los hijos, como se presenta a continuación:

$$
\begin{aligned}
& \text {...tampoco puedo dejar todo aquí [se refiere a San Isidro] y me voy, me voy en la } \\
& \text { tarde, regreso temprano porque el niño también va a la escuela temprano... }
\end{aligned}
$$

Se observó que existen desigualdades en cuanto al apoyo, expectativas y presión por parte de los padres con relación a Flor y su hermano menor, quien actualmente estudia el bachillerato en la comunidad. El padre comenta que en ocasiones Flor le reclama por las malas calificaciones del hermano menor, a lo que el padre asegura que no puede presionarlo, que se conforma con el desempeño académico que el joven actualmente tiene, pues el padre teme que de reclamar al joven, este pueda dejar el bachillerato:

$$
\begin{aligned}
& \text {...pero a veces ve las calificaciones de su hermanito [refiriéndose a Flor]... mira esto } \\
& \text { por qué lo dejas, no estudió [Flor le reclama al padre haciendo referencia a las malas } \\
& \text { notas de su hermano menor]. } \\
& \text { ro no puedo presionarlo le digo, con que lo vaya pasando es con lo que me conformo. } \\
& \text {...ंPor quée, porque si lo presionas tanto va a llegar el momento que sabes qué, ya no } \\
& \text { quiero seguir estudiando y sé que no todos tenemos la misma [capacidad]... }
\end{aligned}
$$

Como se aprecia, en la familia existe un elevado nivel de exigencia hacia Flor y su desempeño académico, mientras que con el hermano menor, los padres son más permisivos y tolerantes.

En cuanto a la educación superior, los participantes en el caso y en especial Flor abordan el tema de la desigualdad de género en el ámbito de las ingenierías. Esta desigualdad muchas veces se traduce en una clasificación de ingenierías por género, para hombres y para mujeres, y en el discurso despectivo de algunos profesores en el entorno de Flor. Respecto al tema de los profesores y la violencia de género que viven las estudiantes de ingeniería, Flor comenta:

$$
\begin{aligned}
& \text {...De hecho actualmente tengo maestros que dicen, se han acostumbrado tanto a tratar } \\
& \text { con hombres, que hacen ese tipo de chistes en el salón y no nos parece...tengo un } \\
& \text { maestro que es horrible, que platicando con otros grupos nos dicen, nunca lo vayan a } \\
& \text { reportar, si se entera que su grupo... se la agarra con nosotros... }
\end{aligned}
$$

Una compañera de Flor, quien al igual que ella cursa la Ingeniería en Bioquímica en la Universidad Tecnológica de Mérida, comenta que incluso en ocasiones los académicos emiten frases ofensivas y machistas. $\mathrm{Al}$ respecto comenta:

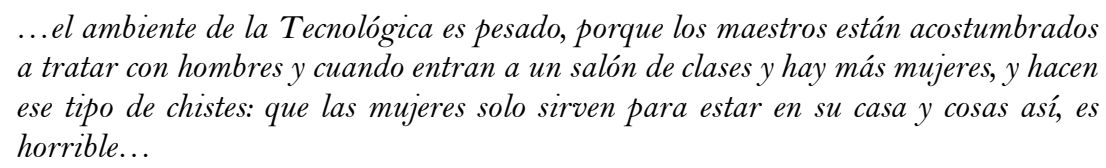

Por otro lado, se observó que internamente existe una clasificación en cuanto a las ingenierías que se imparten en la institución, fenómeno del cual se han percatado los estudiantes, abordando el tema de las ingenierías clasificadas como "para mujeres", incluso algunos profesores tienen percepciones similares. Al respecto María, quien comparte algunas asignaturas con Flor y de igual forma cursa la carrera de Ingeniería Bioquímica en la Universidad Tecnológica de Mérida, relata: 
mayoría de las personas que estudian ingeniería bioquímica o ambiental o química, son mujeres...

Esta es una cuestión crítica que emerge de la observación y de la interacción con Flor, sus amigas y compañeras, siendo una situación que hoy en día viven las estudiantes de la institución e incluso puede estar presente en otras universidades de Yucatán. Con relación a ello, un profesor de la Universidad Tecnológica de Mérida comenta que efectivamente, las mujeres que ingresan a la institución tienen mayor inclinación por ciertas ingenierías, mientras que los hombres por otras muy específicas:

\section{...Eso es una realidad, hoy en día hay mayor participación de mujeres que de hombres en carreras como Ingeniería Ambiental, Bioquímica, Química, Gestión Empresarial y Administración... sin embargo los hombres siguen arrasando en carreras como Ingeniería Civil, Eléctrica, Electrónica, Mecánica y otras por el estilo...}

Esta información contribuye a afirmar que existen ciertas ingenierías, que por su matrícula y la percepción de los participantes son consideradas para mujeres, y otras para hombres, a pesar de que todas se imparten en una institución pública de educación tecnológica.

Estos hallazgos se pueden constatar con los indicadores reportados por la Universidad Tecnológica de Mérida (2017), que en su último informe de rendición de cuentas reporta que existe una mayor participación de mujeres en programas como Ingeniería Ambiental, Ingeniería Bioquímica, Ingeniería Química y la Licenciatura en Administración que se imparten en la institución. Mientas que la participación de hombres es significativamente superior en carreras como Ingeniería Civil, Ingeniería Electrónica, Ingeniería Mecánica e Ingeniería en Sistemas.

\subsection{Calidad de la educación rural}

Un tema que los participantes vincularon de manera automática a la formación académica de Flor fue la calidad de la educación en la comunidad, abordando incluso el tema de la educación científica que se imparte en el municipio, y el paso de Flor por el programa Jóvenes Talentos. Con relación a esta categoría, el hermano menor de Flor comenta:

$$
\begin{aligned}
& \text {...la educación aquí es un poquito mala, porque los maestros no son tan buenos que } \\
& \text { digamos... }
\end{aligned}
$$

Adicionalmente, una amiga de Flor que ha cursado toda la educación básica y media superior en la comunidad afirma que estas carencias se vinculan al hecho de que son de una comunidad rural y la falta de interés de los docentes, quienes muchas veces no se esfuerzan por mejorar su práctica pedagógica al ya haber cumplido con sus objetivos profesionales. Al respecto la joven relata:

$$
\begin{aligned}
& \text { Es que como somos de un pueblo y aquí viven todos los maestros que nos dan en la } \\
& \text { secundaria... son viejitos, ellos ya no salen... que los maestros tienen base y no se } \\
& \text { pueden ir y hay maestros que ya no dan para más y siguen ahí dando clases, muchos } \\
& \text { maestros viven a la vuelta de la escuela... Ya tengo mi base, estoy en mi comunidad, } \\
& \text { gano tanto y ya... }
\end{aligned}
$$

Otra amiga de Flor, complementa la afirmación de la anterior durante una entrevista en San Isidro:

...la educación de aquí es regular, yo siempre he dicho, la educación de Mérida es muy diferente a la de acá...

Los habitantes de la comunidad vinculan la baja calidad de la educación al hecho de vivir en una comunidad rural, lo cual se confirma a través de las afirmaciones del padre de Flor, 
quien como se observa en la siguiente viñeta, comenta que en la comunidad viven personas con mucha capacidad, pero al ser de una comunidad rural tienen mayores retos y dificultades para salir adelante, haciendo énfasis en el "sufrimiento" por el que tienen que pasar para poder sobresalir:

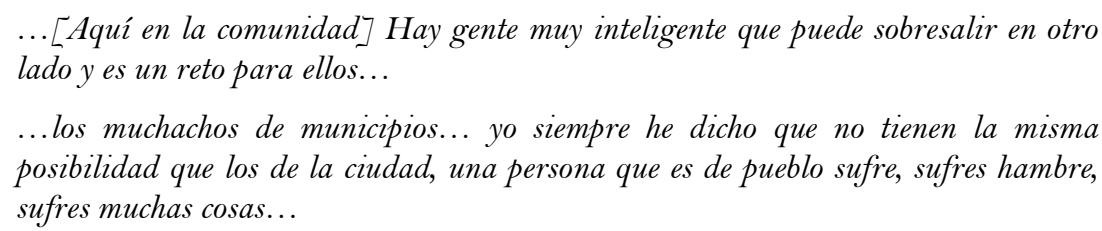

Los participantes hacen énfasis en el hecho de que para las personas que residen en Mérida es más sencillo acceder a una educación de calidad, y otro tipo de actividades que promuevan y fortalezcan la preparación académica de los niños y jóvenes, en comparación con las personas de comunidades rurales.

Con relación a su formación académica inicial, su preparación en ciencia en la comunidad y su paso por Jóvenes Talentos, Flor relata que a través del programa pudo conocer un mundo nuevo respecto al conocimiento científico y tecnológico, pues su comunidad se encuentra rezaga en prácticas, experimentos y actividades de tipo científico en las escuelas. $\mathrm{Al}$ respecto comenta:

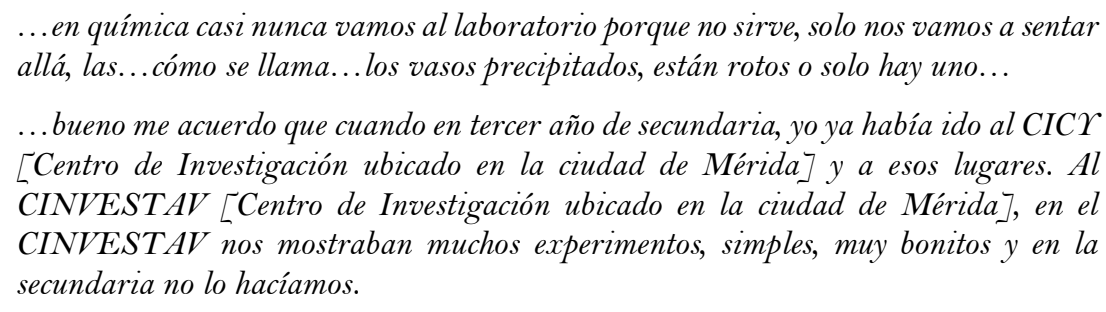

Entonces cuando llegaba al laboratorio les decía, sabes qué, con fab [detergente] y los colores hacen esto y esto y esto, y sale bonito, y de verdad no hacíamos nada de eso. Entonces a nadie le interesa, a nadie le importas... como no hay experimentos, ellos tampoco se interesan en la química, piensan que es pura teoría, no les muestran nada...

Además de despertar su interés por la ciencia y la tecnología, Flor comenta que el programa Jóvenes Talentos influyó de manera significativa en su ingreso a la educación superior, pues afirma:

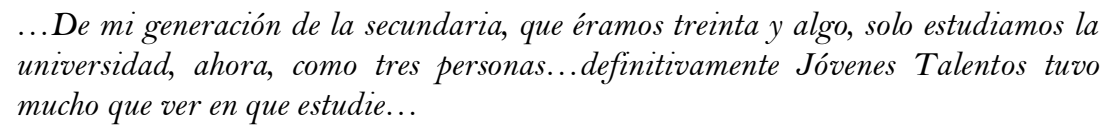

\subsection{Modelos para la formación académica y en investigación}

Se encontró que Flor tuvo modelos a seguir en su familia y en el ámbito de la investigación, los cuales influyeron de manera significativa en su ingreso a la educación superior y específicamente, a una carrera en ciencia y tecnología.

La joven comenta que el primer modelo que tuvo fue el de su tío, una persona con quien se ha identificado desde pequeña y que tuvo que superar ciertas adversidades para poder estudiar una carrera profesional. Vale la pena señalar que dicho esfuerzo y estudios influyeron en que actualmente el tío sea una persona estable económicamente, lo que ha influido en el interés de Flor por estudiar la educación superior, pues comenta: 
pensaba él era mi tío el rico, porque mi tío era el que tenía carro, el que tenía casa propia, trabajo y todo eso... si estudias una carrera, vas a tener una mejor calidad de vida...

Finalmente, se encontró que uno de los modelos más fuertes de Flor fue un investigador del Centro de Investigaciones Regionales de la Universidad Autónoma de Yucatán, quien la motivó a continuar con una carrera en ciencia y tecnología, esto durante su estancia en Jóvenes Talentos. La joven comenta que la motivación fue aún más fuerte porque el investigador, al igual que ella, provenía de una comunidad rural y logró estudiar una carrera profesional, para posteriormente trabajar en el Centro de Investigaciones. Flor comentó al respecto:

...para cuando yo estaba en Jóvenes Talentos... el investigador fue una persona muy importante y fue la persona que más se interesó en mí, cuando me preguntó qué quieres estudiar. Osea fue una persona que se acercó a mí, que me apoyó, que me decía, tú puedes Flor...porque para mí fue muy difícil estudiar allá, viajar hasta allá, es mucho tiempo, es mucho dinero y a veces mis papás me decían no tengo y él siempre me decía, tú puedes...

...en un futuro, sí me veo, no como él, pero, no mejor, bueno sí mejor [ríe $]$...

... Me gustó su historia de cómo estudió su universidad y que con sus prácticas o residencia, fue que consiguió trabajo en el Centro de Investigaciones...

...Por una parte me impulsó porque digo, si él pudo siendo igual de otra comunidad, yo también puedo...

Estos hallazgos contrastan con resultados de estudios realizados en Asia y Europa (Badri, et al., 2016; Osborne, Simon y Collins, 2003), en los que se ha encontrado que hoy en día existe una falta total de modelos contemporáneos en ciencias. De igual manera, el tema de los modelos en el caso de Flor responde a las necesidades planteadas en los trabajos citados acerca de la urgencia que hoy en día existe por hablar menos del método científico, los grandes hombres de ciencia y hablar más a los jóvenes acerca de lo que hace un biólogo o un físico de manera cotidiana.

Por otro lado, el tema de los modelos en ciencias que emerge del caso de Flor es consistente con el estudio de Estrada-Hollenbeck, et al. (2011, p. 2), quienes afirman que "en el ambiente académico, los agentes influyentes son representantes o miembros de la comunidad científica académica. Los objetivos de influencia son potenciales y actuales estudiantes de ciencias" y adicionalmente, afirman que dichos agentes tienen influencia directa en que los estudiantes permanezcan en las ciencias, convirtiéndose en un futuro en científicos profesionales.

\section{Conclusiones}

Esta investigación tuvo como propósito comprender los retos, las experiencias y las cuestiones críticas de una joven maya durante su incursión en la educación superior tecnológica, la ciencia y la tecnología. Para ello se llevó a cabo un estudio de caso cualitativo, diseño que permitió comprender a profundidad las cuestiones críticas que ha experimentado el caso durante la educación superior, así como en su incursión en el ámbito científico y tecnológico. Si bien los resultados no son generalizables, sirven como base para explorar las cuestiones críticas que viven otras jóvenes de la región maya de México que estudian la educación superior, ciencia y tecnología. 
En el estudio se encontró que si bien se ha incrementado la participación de las mujeres en el ámbito de la ciencia y la tecnología, particularmente en el área de las ciencias naturales (Poczatková y Křibíková, 2017), se considera que debido a la modernidad y factores de segregación interna en la ciencia, las jóvenes no consideran disciplinas vinculadas a las ingenierías y las tecnologías como opciones de carreras profesionales, pues son clasificadas como carreras para varones.

A nivel de caso se puede afirmar que las jóvenes de comunidades rurales que se involucran en el ámbito de la ciencia y tecnología, desarrollan un fuerte sentido de resiliencia ante las situaciones que afrontan durante su formación, lo cual es consistente con el estudio de Aguiar y Acle (2012). Esta resiliencia se configura a través de múltiples factores que afrontan como la migración, desconocimiento del contexto urbano, requerimiento de conocimientos especializados en ciencia y tecnología, segregación del sistema educativo formal, pobreza, entre otros.

De igual forma se observó que los modelos académicos, como pueden ser amigos y familiares, así como el apoyo familiar y comunitario representan la base sobre la que se construye esta resiliencia. Se recomienda establecer estrategias que promuevan una mayor participación de niñas, jóvenes y mujeres en el ámbito científico, con énfasis en la población maya de zonas rurales, así como actividades que propicien la concientización en temas de equidad de género en los sectores mencionados.

Desde el estudio de caso se puede concluir que en la comunidad académica de Yucatán, particularmente en la educación superior y tecnológica, existe una clasificación de carreras profesionales para hombres y mujeres, que van más allá de los estereotipos ya estudiados como la idea de que las ingenierías son para hombres y las humanidades y ciencias social son para mujeres. Al respecto se encontró que dentro del ámbito de las ingenierías se construye una clasificación, no escrita o visibilizada, en cuanto a que existen ingenierías "duras" como mecánica, eléctrica o sistemas computacionales que son para hombres; e ingenierías "blandas" como energías renovables, administración o química para mujeres. Por lo anterior, se recomienda el desarrollo de estudios posteriores que exploren las desigualdades y estereotipos de género que existen en el ámbito de las ingenierías en Yucatán.

Finalmente, se recomienda a las autoridades en general establecer esquemas de apoyos económicos, de vivienda y de alimentos para los jóvenes de comunidades rurales que migran a los sectores urbanos con el fin de estudiar la educación superior, pues además de los retos académicos y sociales que los jóvenes enfrentan, muchos afrontan fuertes necesidades económicas para continuar con su preparación académica en sectores alejados de sus comunidades de origen.

\section{Referencias}

Aguiar, E. y Acle, G. (2012). Resiliencia, factores de riesgo y protección en adolescentes mayas de Yucatán: Elementos para favorecer la adaptación escolar. Acta Colombiana de Psicología, $15(2), 53-64$.

Asociación Nacional de Universidades e Instituciones de Educación Superior. (2019). Anuario estadístico de educación superior 2018-2019. ANUIES.

Badri, M., Alnuaimi, A., Mohaidat, J., Al Rashedi, A., Yang, G. y Al Mazroui, K. (2016). My science class and expected career choices, a structural equation model of determinants 
involving Abu Dhabi high school students. International Journal of STEM Education, 12(3), 1-21. https://doi.org/10.1186/s40594-016-0045-0

Cárdenas, M. (2015). La participación de las mujeres investigadoras en México. Revista Investigación Administrativa, 64, 116-135.

CONACYT. (2017). Reglamento del sistema nacional de investigadores. CONACYT

CONACYT. (2018). Archivo del sistema nacional de investigadores. Investigadores vigentes 2018. CONACYT

Consejo Estatal de Población. (2020). Indicadores sobre nacimientos y tasas de fecundidad. Gobierno del Estado de Yucatán.

Denzin, N. y Lincoln, Y. (2005). The Sage handbook of qualitative research. Sage.

Di Cesare, M. (2014). Women, marginalization, and vulnerability: Introduction. Genius, $7 O(2), 1-6$.

Estrada-Hollenbeck, M., Woodcock, A., Hernandez, P. y Schultz, P. W. (2011). Toward a model of social influence that explains minority student integration into the scientific community. Journal of Educational Psychology, 103(1), 206-222. https://doi.org/10.1037/a0020743

Gobierno del Estado de Yucatán. (2019). Plan estatal de desarrollo de Yucatán 2018-2024. Gobierno del Estado de Yucatán.

González González, R. J. y Cisneros-Cohernour, E. J. (2020). Justicia social e inequidad en la formación científica y tecnológica de jóvenes rurales en la región maya de México: El caso de Mex. Revista Internacional de Educación para la Justicia Social, 9(1), 19-39. https://doi.org/10.15366/riejs2020.9.1.001.

Instituto Nacional de Estadística y Geografía. (2015). Encuesta intercensal 2015. Principales resultados. Instituto Nacional de Estadística y Geografía.

Instituto Tecnológico de Mérida. (2017). Informe de rendición de cuentas 2017. Instituto Tecnológico de Mérida.

Izquierdo, I. y Atristan, M. (2019). Experiencias de investigadoras en su ingreso, promoción y permanencia en el sistema nacional de investigadores: Tensiones y estrategias identitarias. Revista de Investigación Educativa de la REDIECH, 1O(18), 127-142.

https://doi.org/10.33010/ie_rie_rediech.v10i18.466

Jiménez Izquierdo, Y., Magaña Medina, D. E. y Valdés Cuervo, A. A. (2019). Interés de estudiantes hacia carreras universitarias en ingeniería. Educación y Ciencia, 8(51), 83-88.

Kawakita, J. (1977). Ido daigaku or free campus university. Research in Higher Education-Daigaku Ronshu, 5, 91-102.

Kumar, T. y Yadav, K. (2018). Women's education and political participation. International Journal of Advanced Education and Research, 3(6), 65-71. https://doi.org/10.22271/educatin.2018.v3.i6.15

Mijangos, J. C., Canto, P. J. y Cisneros, E. J. (2009). Introducción. En J. C. Mijangos (Ed.), La lucha contra el rezago educativo. El caso de los mayas en rucatán (pp. 9-44). Unas Letras Industria Editorial.

ONU Mujeres. (2017). El progreso de las mujeres en América Latina y el Caribe 2017. AECID.

Osborne, J., Simon, S. y Collins, S. (2003). Attitudes towards science: A review of the literature and its implications, International Journal of Science Education, 25(9), 1049-1079.

https://doi.org/10.1080/0950069032000032199 
Patton, M. Q. (2015). Qualitative research y evaluation methods: integrating theory and practice. Sage.

Poczatková, B. y Křibíková, P. (2017). Gender inequality in the field of science and research. Journal of International Studies, 1O(1), 267-276. https://doi.org/10.14254/2071-8330.2017/10-1/19

Remes, M. J. y Cervera, C. (2016). El techo de cristal en las universidades. Jóvenes en la ciencia, Revista de divulgación de la ciencia, 2(1), 1767-1771.

Rodríguez, A.C. (2008). Las mujeres en la academia nacional de medicina de México: Análisis de su inserción y ubicación en la élite médica. Gaceta Médica Mexicana, 144(3), 265-270.

Rodríguez, C. (2016). El sistema nacional de investigadores en números. Foro Consultivo, Científico y Tecnológico.

Stake, R. (1998). Investigación con estudio de casos. Morata.

Stake, R. (2010). Qualitative Research, Studying How Things Work. Guilford Press.

UNESCO. (2003). Gender and education for all: The leap to equality. UNESCO.

\section{Breve CV de los/as autores/as}

\section{Roger J. González González}

Licenciado en Educación, Maestro en Investigación Educativa y candidato a Doctor en Ciencias Sociales por la Universidad Autónoma de Yucatán. Es coautor de dos libros enfocados en el desarrollo de competencias digitales de la editorial Pearson, así como de diversos capítulos de libro y ponencias en congresos nacionales y en el extranjero. Tiene experiencia docente a nivel licenciatura y posgrado, sus áreas de investigación se centran en la justicia social y educativa, la formación de investigadores, la evaluación de programas y la tecnología educativa. ORCID ID: https://orcid.org/O000-0003-2876-3539. Email: rogr.gonzalez@gmail.com

\section{Edith J. Cisneros-Cohernour}

Doctora en Evaluación, Administración y Educación Superior por la Universidad de Illinois at Urbana-Champaign. Su investigación se centra en el mejoramiento de la calidad de la educación en las organizaciones educativas, especialmente en las áreas de evaluación, desarrollo profesional y organizacional y los aspectos éticos en evaluación e investigación. Actualmente es Jefa de la Unidad de Posgrado e Investigación en la Facultad de Educación de la Universidad Autónoma de Yucatán y pertenece al nivel dos del Sistema Nacional de Investigadores del CONACYT. ORCID ID: https://orcid.org/O000-0003-2319-1519. Email: ecohernour@gmail.com

\section{Galo E. López Gamboa}

Doctor en Investigación en Humanidades y Educación por la Universidad de Castilla-La Mancha, España. Licenciado en Educación y Maestro en Investigación Educativa por la Universidad Autónoma de Yucatán (UADY). Es profesor en la Facultad de Educación de la UADY y Coordinador de la Licenciatura en Educación en la misma. Miembro del Consejo Mexicano de Investigación Educativa. Ha sido asesor y evaluador de proyectos de formación para la Secretaría de Innovación, Investigación y Educación Superior del Estado de Yucatán. ORCID ID: https://orcid.org/O000-0001-5581-7489. Email: lopegam@gmail.com 